\title{
A Mechanism Study of NMDAR1 in A Rat Alzheimer Disease (AD) Model
}

\author{
Tianzhong Peng ${ }^{1}$, Xuedi Huang ${ }^{2}$, Suifa Hu${ }^{1}$, Gui Xie ${ }^{1}$, Cheng Zhou ${ }^{1}$, Jia Xiong ${ }^{1}$, Rui Liu ${ }^{3 *}$. \\ ${ }^{1}$ Department of Neurology, Nanchang Hongdu Hospital of Traditional Chinese Medicine; ${ }^{2}$ Department of Clinical \\ Pharmacy, the Second Hospital of Nanchang; ${ }^{3}$ Basic Medical College, Jiangxi University of Traditional Chinese \\ Medicine.
}

\begin{abstract}
This study aimed to investigate the expression and mechanism of $N$ - methyl $-D$ - aspartate receptor 1 (NMDARl) in the pathogenesis of Alzheimer disease (AD). Eighty adult Wistar rats were randomly divided into 4 groups $(n=20$ each) to receive an injection of $0,5,7$ and $10 \mu \mathrm{l}$ of $1 \mu \mathrm{g} / \mu \mathrm{l}$ amyloid- $\beta 42\left(A \beta_{1-42}\right)$ in the hippocampus. Twenty rats in normal control group were injected with equal volume of saline. After 10 days, the hippocampus was isolated from 5 randomly selected rats in each group. The NMDARI protein and mRNA expression was determined by immunohistochemical staining and qRT-PCR. The aquaporin-1 (AQP-1) mRNA expression was also measured by qRT-PCR. We found that both NMDAR1 and AQP-1 expression in A $\beta_{1-42}$ groups was increased in a dose-dependent manner. NMDARl and AQP-1 expression in 7 and $10 \mu \mathrm{l} \beta_{1-42}$ groups was significantly higher compared with $0 \mu l$ $A \beta_{1-42}$ group $(P<0.01)$. Further, the $10 \mu \mathrm{l} A \beta_{1-42}$ group was randomly divided into 3 subgroups: $A D-N M D A, A D-$ $M K-801$, and AD-Ctrl subgroup, which was given an intraperitoneal injection of NMDAR agonist NMDA, NMDAR antagonist $M K-801$ and saline, respectively. The relative $A P Q-1$ expression in each subgroup was determined by qRT-PCR and Western blot analysis after $24 \mathrm{~h}$. The AQP-1 expression was significantly decreased in AD-MK-801 group $(P<0.05)$, but was markedly increased in AD-NMDA group when compared with AD-Ctrl group $(P<0.01)$. Our study suggested that expression abnormity of NMDAR1 is involved in the pathogenesis of AD. NMDAR1 might regulate the pathogenic process through stimulating the expression of $A Q P-1$.
\end{abstract}

Key words: NMDAR1, Alzheimer disease, aquaporin, AQP-1

\footnotetext{
*Author for correspondence: omvm2244011@126.com
} 


\section{INTRODUCTION}

Alzheimer's disease (AD) is a progressive neurodegenerative disease with insidious onset. With the aging of the global population, the incidence rate of AD is gradually increasing, leading to a patient population of approximately thirty million worldwide $[1,2]$. The main pathological features of $A D$ include neuronal loss, amyloid- $\beta$ deposition, and neurofibrillary tangles in nerve cells. Patients develop memory and cognitive impairment in early stages, and suffer from severe intelligent recession in advanced stages [3]. The pathogenesis of AD is not yet fully elucidated and thus relevant researches have significant clinical importance.

Studies have shown that brain damage is usually accompanied by massive release of glutamate, which exerts persistent neurotoxic effects by binding to the receptors $[4$, 5]. N- methyl -D- aspartate receptor (NMDAR)-mediated glutamate excitotoxicity activates relevant signaling pathways by stimulating $\mathrm{Ca}^{2+}$ influx. The functional subtype NMDAR1 is essential for the activity of NMDAR-receptor complexes [6, 7]. In this study, we investigated the mechanism of NMDAR1 in an AD rat model in order to provide an experimental basis for the pathogenesis of the disease.

Aquaporin (AQP) is a group of membrane channel proteins that regulate the permeability of water and other small molecules. Normal expression of AQP in the brain tissues plays an important role for the local water transport [8]. Moreover, as the aquaporins in the central nervous system, AQP is crucial for the maintenance of normal ion concentrations in the brain tissue [9]. Balance disorders of ions and water molecules have been detected in the brian tissues of $\mathrm{AD}$ patients [10]. Hoshi et al. have found that the number of AQP-1-positive cells in AD patients is significantly higher than that in normal brain tissues [11], suggesting that abnormal expression of AQP-1 might be associated with the occurrence of AD. In this study, the expression and mechanism of NMDAR1 in an AD rat model was determined by real-time PCR and immunohistochemical analyses. The association between NMDAR1 and AQP-1 was also investigated to explore the potential function of both proteins in the pathogenesis of $\mathrm{AD}$.

\section{MATERIAL AND METHODS}

\section{Reagents and instruments}

Wistar rats were purchased from Fukang Biotech. (Beijing, China). Amyloid- $\beta$ 1-42 $\left(\mathrm{A} \beta_{1-42}\right)$ was purchased from Sigma (St. Louis, MO, USA) and was prepared into 1 $\mu \mathrm{g} / \mu \mathrm{l}$ stock. MK-801 (dizocilpine) and N-methyl-D-aspartate (NMDA) were purchased from Sigma. Total RNA extraction kit was purchased from Exiqon (Catalog No. 300110, Vedbaek, Denmark). Primers were synthesized by Shenggong Biotech. (Shanghai, China). PrimeScrip RT reagent kit and SYBR Fast qPCR Mix were purchased from Takara (Tokyo, Japan). APQ-1 and NMDAR1 monoclonal antibodies were purchased from Santa Cruz (Santa Cruz, CA, USA). Horseradish peroxidase (HRP)-labeled secondary antibody was purchased from Boster Biotech. (Wuhan, China). Immunohistochemical kit was purchased from Roche (Mannheim, Germany). Nucleic acid was quantified with a Nanodrop 2000 from Thermo Fisher Scientific (San Jose, CA, USA). Quantitative real-time PCR (qRT-PCR) was performed using an Applied Biosystems 7500 fluorescence system. Tissue sections were observed with an Olympus BX53 optical microscope.

\section{Construction of AD rat model and grouping}

This study was approved by the Research Ethics Committee of Jiangxi University at the Traditional Chinese Medicine. The AD rat model was constructed as previously 
described [12, 13]. Briefly, 80 healthy adult female Wistar rats weighing $200 \pm 20 \mathrm{~g}$ were randomly divided into 4 model groups $\left(0,5,7\right.$, and $10 \mu \mathrm{A} \beta_{1-42}, \mathrm{n}=20$ each). $\mathrm{A} \beta_{1-42}$ solution $(1 \mu \mathrm{g} / \mu \mathrm{l})$ was prepared with sterile PBS and incubated at $37{ }^{\circ} \mathrm{C}$ for 72 $\mathrm{h}$ until aggregation was observed. All rats were anesthetized with $10 \%$ chloral hydrate. The head was placed on the brain locator. The skin around the skull was disinfected and a small incision was cut. A $0.8-\mathrm{mm}$ hole was drilled at the hippocampus CAI area with a medical micro drill, and $0,5,7$, and $10 \mu \mathrm{A} \beta_{1-42}$ was injected, respectively. The skin was sutured after the operation. Twenty rats in normal control group were injected with equal volume of sterile saline. All rats were given intramuscular antibiotics injection and raised in standard animal cages at 18 $25^{\circ} \mathrm{C}$ with free access to water and food throughout the experiment. At 10 days after the surgery, 5 rats were randomly selected from each group. The hippocampus was isolated from each rat and subjected to subsequent analyses.

Further, rats in the $10 \mu \mathrm{l} A \beta_{1-42}$ group was randomly divided into 3 subgroups ( $\mathrm{n}=5$ each): AD-NMDA, AD-MK-801, and AD-Ctrl group, which were given an intraperitoneal injection of NMDAR agonist NMDA $(20 \mathrm{mg} / \mathrm{kg})$, NMDAR antagonist MK-801 (1 mg/kg), and saline, respectively. After $24 \mathrm{~h}$, the hippocampus was isolated and stored in liquid nitrogen.

\section{Immunohistochemical staining}

Tissue samples were fixed with $4 \%$ paraformaldehyde, dehydrated with serial ethanol $(70,80,90$, and $100 \%$ ethanol), embedded in paraffin, cut into 4-um sections, and mounted in neutral gum. The sections were deparaffinized 3 times with xylene, and rehydrated through serial ethanol (100, 90, 80, and 70\% ethanol) and Tris-buffered saline. The sections were heated in antigen retrieval solution in a pressure cooker for $5 \mathrm{~min}$, and incubated in 3\% hydrogen peroxide for $8 \mathrm{~min}$ to block the endogenous peroxidases. The sections were then incubated with mouse anti-rat NMDAR1 monoclonal antibody (1:500 dilution) at room temperature for $120 \mathrm{~min}$. The sections were rinsed and incubated with rabbit anti-mouse secondary antibody (1:1000 dilution) at room temperature for $120 \mathrm{~min}$, followed by DAB color development for $6 \mathrm{~min}$. Brown positive-stained cells were detected using a light microscope. Data was analyzed using the Image-ProPlus software. Specifically, 5 areas of interest (AOI) were randomly selected, and the mean integrated option density (IOD) and the effective area was measured. The expression level of NMDAR1 was calculated as the mean density $=$ IOD/area.

\section{qRT-PCR analysis}

The collected tissue sample was homogenized in liquid nitrogen. Total RNA was extracted using total RNA extraction reagent and quantified using a Qubit fluorometer at $260 \mathrm{~nm}$. RNA was reverse transcribed into cDNA using the reverse transcription kit following the manufacture's instruction. Primers were designed based on the sequences of target genes (NMDAR1, NM_008169.3; AQP-1, NM_007472; GAPDH, NM_008085) downloaded form the NCBI database and synthesized by Shenggong Biotech.: NMDAR1 (NM_008169.3) Forward: CTGCGACCCCAAGATTGTCAA, Reverse: TATTGGCCTGGTTTACTGCCT; AQP-1 Forward: AGGCTTCAATTACCCACTGGA, Reverse: CTTTGGGCCAGAGTAGCGAT; and GAPDH Forward: AATGGATTTGGACGCATTGGT, Reverse: TTTGCACTGGTACGTGTTGAT. The $\mathrm{Ct}$ value of each gene was measured and the relative expression of target gene was calculated by the double $\triangle \mathrm{Ct}$ method [14] using the following formulas: $\Delta \mathrm{CT}$ $=\mathrm{CT}$ (Target gene) $-\mathrm{CT}$ (internal reference), and $\Delta \Delta \mathrm{CT}=\Delta \mathrm{CT}$ (sample) $-\Delta \mathrm{CT}$ 
(contro1), and the relative expression level of target gene $=2-\Delta \Delta$ CT (the relative expression of control group: $\left.2^{0}=1\right)$. The reaction system $(20 \mu \mathrm{l})$ was prepared: $2 \times$ SYBR Fast qPCR Mix: $10 \mu \mathrm{l}$; PCR forward / reverse primer $(10 \mathrm{mM}): 0.8 \mu \mathrm{l} ; 50 \times$ ROX reference dye II: $0.4 \mu \mathrm{l}$; cDNA template: $2 \mu \mathrm{l}$, and PCR was performed using the following program: denaturation at $95{ }^{\circ} \mathrm{C}$ for $30 \mathrm{~s}$, followed by 40 cycles of 95 ${ }^{\circ} \mathrm{C}$ for $3 \mathrm{~s}$ and $60{ }^{\circ} \mathrm{C}$ for $13 \mathrm{~s}$. The experiment was repeated three times.

\section{Western blot analysis}

Tissue samples were homogenized in liquid nitrogen and lysed with RIPA lysis buffer and protease inhibitor on ice for $30 \mathrm{~min}$, followed by ultrasonic lysis. The lysate was centrifuged at $13000 \mathrm{r} / \mathrm{min}$ at $4{ }^{\circ} \mathrm{C}$ for $20 \mathrm{~min}$. The supernatant was transferred to a new EP tube, and the concentration of protein was measured using the BCA protein assay kit following the manufacture's instruction. Equal amounts of total protein $(10 \mu \mathrm{g})$ were separated by SDS-PAGE electrophoresis and transferred to polyvinylidene difluoride membranes at $100 \mathrm{~V}$ for $1 \mathrm{~h}$. The membrane was blocked in TBS buffer containing 5\% skim milk and $0.1 \%$ Tween 20 at room temperature for $1 \mathrm{~h}$, and incubated with mouse anti-rat AQP-1 antibody (1:5000) at room temperature for $2 \mathrm{~h}$. the membrane was washed 3 times with TBST for 5 min each, and incubated with HPR-labeled rabbit anti-mouse secondary antibody (1:10000) at room temperature for $1 \mathrm{~h}$. The membranes were washed 3 times with TBST for 5 min each and incubated with fixing and developing reagents for ECL detection. The intensity of bands was detected by a Molecular Imager ${ }^{\circledR}$ ChemiDocTM XRS System (Bio-Rad Laboratories). The gray value of bands was analyzed by Image Lab 2.0 software (Bio-Rad Laboratories). The relative expression level of target protein was calculated as the ratio of gray value of target protein to that of the internal control.

\section{Statistical analysis}

All data were expressed as mean \pm standard deviation, and analyzed by SPSS11.5 software (SPSS Inc., Chicago, IL, USA). The difference between groups was analyzed by t-tests. $\mathrm{P}$ values smaller than 0.05 were considered statistically significant.

\section{RESULTS}

\section{Comparison of NMDAR1 protein and mRNA expression in groups receiving different doses of $A \beta_{1-42}$}

The NMDAR1-positive area in hippocampus tissue in all groups was determined by immunohistochemical staining (Figure 1). As shown in Figure 2a, the mean density in 0 and $5 \mu \mathrm{A} \mathrm{A} \beta_{1-42}$ group $(0.31 \pm 0.06$ and $0.45 \pm 0.04$, respectively) was comparable to that in control group $(0.29 \pm 0.01, \mathrm{P}>0.05)$. The mean density in 7 and $10 \mu \mathrm{A} \beta_{1}$ 42 group $(0.67 \pm 0.07$ and $0.99 \pm 0.10$, respectively) was significantly higher compared with $0 \mu \mathrm{A} \beta_{1-42}$ group $(\mathrm{P}<0.05)$, suggesting that the expression of NMDAR1 in rats was increased in a $A \beta_{1-42}$ dose-dependent manner. 

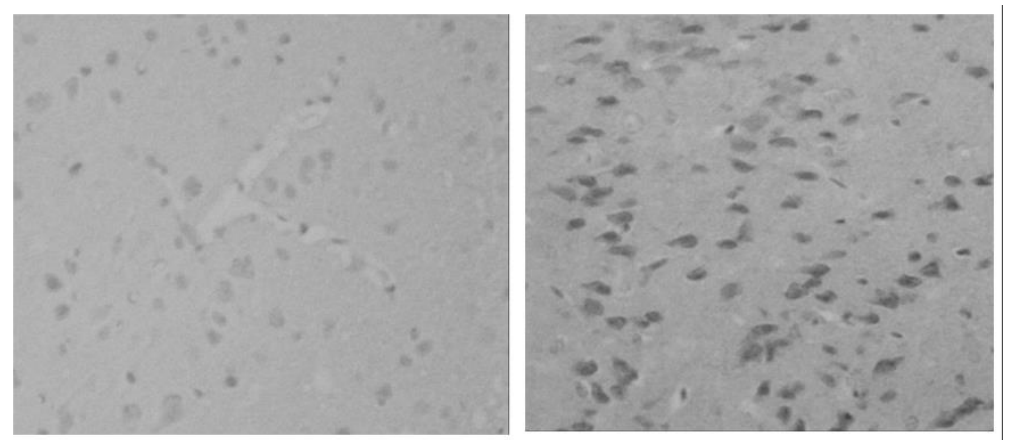

Figure 1. Representative immunohistochemical images (100x) showing NMDAR1-positive area in control (left) and $10 \mu \mathrm{l} A \beta_{1-42}$ group (right).
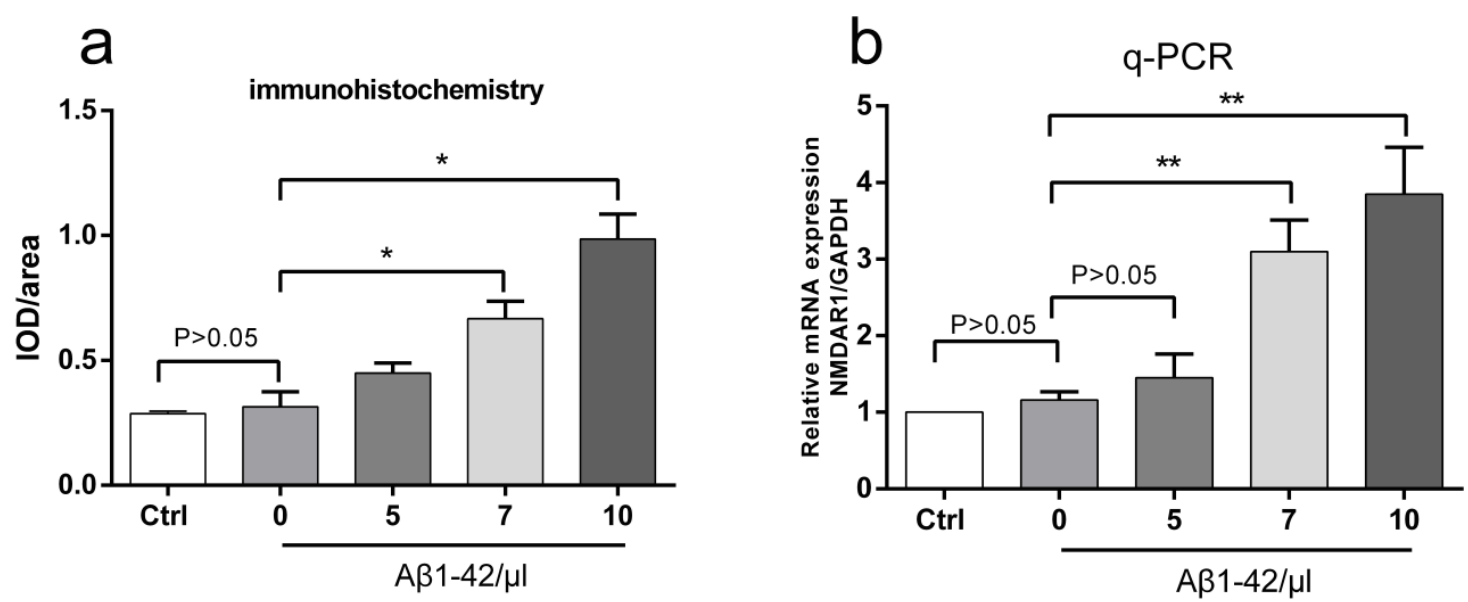

Figure 2. Comparison of NMDAR1 protein and mRNA expression in different groups of AD rats. (a) Comparison of NMDAR1-positive area in different groups by immunochemical analyses. (b) qRT-PCR analyses of NMDAR1 mRNA expression in different $\mathrm{A} \beta_{1-42}$ groups. *, $\mathrm{P}<0.05$ compared with $0 \mu \mathrm{l} \beta_{1-42}$ group; **, $\mathrm{P}<0.01$ compared with $0 \mu \mathrm{A} \mathrm{A} \beta_{1-42}$ group.

The relative expression of NMDAR1 mRNA among all groups was further compared by qRT-PCR. As shown in Figure $2 b$, there was no significant difference in the relative NMDAR1 mRNA expression among control, 0 and $5 \mu \mathrm{A} \beta_{1-42}$ groups $(\mathrm{P}>0.05)$. The NMDAR1 mRNA expression in both 7 and $10 \mu \mathrm{A} \beta_{1-42}$ group was significantly higher compared with $0 \mu \mathrm{A} \beta_{1-42}$ group $(\mathrm{P}<0.01)$, suggesting that NMDAR 1 mRNA expression was also gradually increased with higher dose of $A \beta_{1-}$ 42 .

Comparison of AQP-1 expression in groups receiving different doses of $A \beta_{1-42}$ The relative expression level of AQP-1 in hippocampus tissues in all groups was determined by qRT-PCR. As shown in Figure 3, AQP-1 mRNA expression in 5, 7 and $10 \mu \mathrm{l} A \beta_{1-42}$ groups was significantly higher compared with $0 \mu \mathrm{l} A \beta_{1-42}$ group (P $<0.05$ or 0.01 ), suggesting that AQP-1 expression in hippocampus tissues was increased in a $A \beta_{1-42}$ dose-dependent manner. 


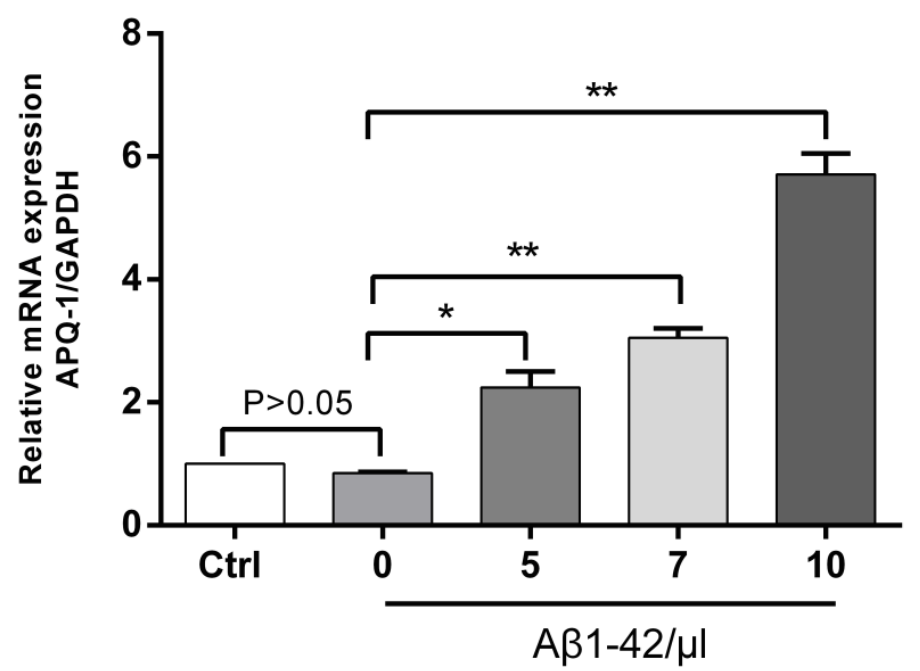

Figure 3. qRT-PCR analyses of AQP-1 mRNA expression in the hippocampus of AD rats in different groups. *, $\mathrm{P}$ $<0.05$ compared with $0 \mu \mathrm{A} A \beta_{1-42}$ group; **, $\mathrm{P}<0.01$ compared with $0 \mu \mathrm{l} A \beta_{1-42}$ group.

\section{Association between NMDAR1 and APQ-1 expression}

To study the association between NMDAR1 and APQ-1 expression in hippocampus tissues of $\mathrm{AD}$ rats, the $10 \mu \mathrm{A} \beta_{1-42}$ group was randomly divided into 3 subgroups: AD-NMDA, AD-MK-801, and AD-Ctrl subgroup, which was given an intraperitoneal injection of NMDAR agonists NMDA, NMDAR antagonist MK-801 and saline, respectively. The relative APQ- 1 mRNA expression in the 3 subgroups was compared by qRT-PCR after $24 \mathrm{~h}$. As shown in Figure 4, APQ-1 mRNA expression in AD-MK-801 group was decreased by 17.05\% compared with AD-Ctrl group $(\mathrm{P}<0.05)$, whereas APQ-1 mRNA expression in AD-NMDA was significantly increased $(\mathrm{P}<0.01)$.

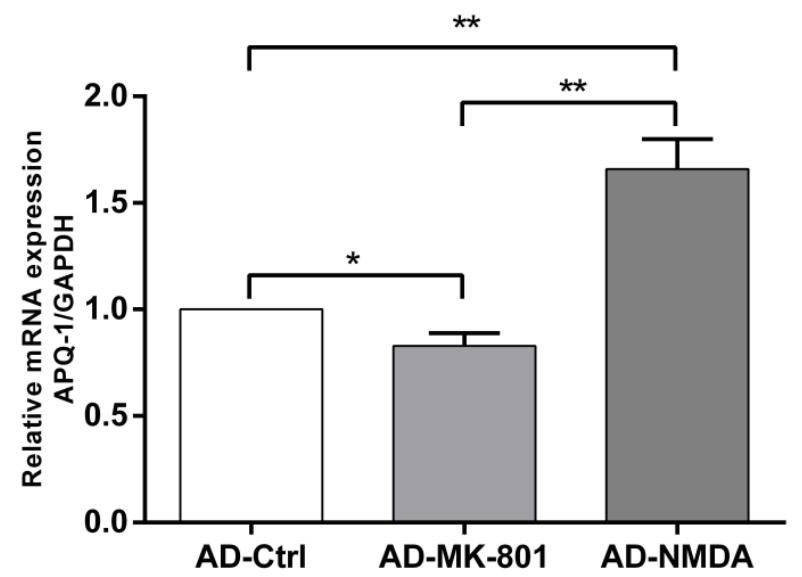

Figure 4. qRT-PCR analyses of relative AQP-1 mRNA expression in the three subgroups (AD-NMDA, AD-MK801, and AD-Ctrl subgroups) of the $10 \mu 1 \mathrm{~A} \beta_{1-42}$ group. *, $\mathrm{P}<0.05$ and ${ }^{* *}, \mathrm{P}<0.01$.

Consistent with the results in qRT-PCR analysis, Western blot showed that the expression of APQ-1 protein in AD-NMDA group was the highest, followed by ADCtrl $(\mathrm{P}<0.01)$. The lowest APQ-1 expression was observed in AD-MK-801 group $(0.44 \pm 0.06)$, which was significantly lower compared with AD-Ctrl $(\mathrm{P}<0.05)$.

\section{DISCUSSION}


Signal transduction plays an important role in the pathogenesis of $\mathrm{AD}$. As the excitatory neurotransmitter in the central nervous system, glutamic acid exerts excitatory effects on neurons through NMDAR, one of the glutamate receptors. There are two major subtypes of NMDAR including the functional subtype NMDAR1, and the regulatory subtype NMDAR2. Studies have found that NMDAR can regulate the synaptic growth, development and plasticity through changing the ratio of NMDAR1/NMDAR2, NO-mediated enzyme and the ion channel [15, 16], and ultimately regulate signal transmission between synapses. Therefore, studies on NMDAR1 expression might provide insights into the role of NMDAR1 in the pathogenesis of AD.

An appropriate animal model of $\mathrm{AD}$ is an essential basis for relevant studies. Currently, there are several construction methods of $\mathrm{AD}$ animal models including $\beta$ amyloid-based method, amyloid precursor protein (APP) transgenic animal, and hippocampal fimbria-fornix damage model. "A $\beta$ hypothesis" is currently considered as one of the major pathogenic mechanisms of $\mathrm{AD}$, and $\mathrm{A} \beta$ accumulation is the key mechanism for the occurrence and development of AD [17, 18]. APP can be cleaved into $A \beta$ under the catalysis of $\beta$ - and $\gamma$ - secretase. While $A \beta 40$ is the normal substances in the cerebrospinal fluid, $A \beta 42$ is known as the key factor inducing the formation of amyloid plaques in the brain. In this study, an AD animal model was constructed by injection of $A \beta_{1-42}$ in the hippocampus of rats. In our preliminary studies, $\mathrm{A} \beta$ antibodies were detected in the plasma of these rats. Significant deficiency in spatial memory of these rats was also observed in Morris water maze test, confirming the success of the model construction.

In this study, the expression of NMDAR1 in tissues was quantified by immunohistochemical staining. Results showed that the NMDAR1 expression in 0 , 5, 7 and $10 \mu \mathrm{A} \beta_{1-42}$ group was increased in a dose-dependent manner. Consistent with immunohistochemical analyses, qRT-PCR also demonstrated a steady increase in NMDAR1 mRNA expression in 0, 5, 7 and $10 \mu \mathrm{A} \beta_{1-42}$ groups. Moreover, the NMDAR1 mRNA expression in both 7 and $10 \mu \mathrm{A} \beta_{1-42}$ group was significantly higher compared with $0 \mu \mathrm{l} A \beta_{1-42}$ group $(\mathrm{P}<0.01)$. These results implied that NMDAR1 expression was positively correlated with the dose of injected $A \beta_{1-42}$ and suggested that the aggravated injury in cognitive function might be associated with the sustained increase in NMDAR1 expression. Similar finding has been previously reported, in which NMDAR1 expression was positively correlated with the degree of traumatic brain edema [19].

AQP-1 in the brain tissue is primarily involved in the formation of cerebrospinal fluid and the regulation of the balance of water and ion [20]. Studies have found that $\mathrm{A} \beta$ deposition may stimulate abnormal expression of AQP-1. During the development of brain injury-induced cerebral edema, AQP-1 changes the permeability of cerebrospinal fluid by regulating the transport of water molecule [10]. AQP-1 functional abnormity might become the key factor for several diseases of the central nervous system that are characterized by brain edema. To date, it remains a question whether AQP-1 is involved in the process of nerve damage in $\mathrm{AD}$ patients. In this study, the expression of AQP-1 mRNA in different groups was compared by qRT-PCR. It was found that AQP-1 expression in 0, 5, 7 and $10 \mu \mathrm{A} \beta_{1 \text { - }}$ 42 group was gradually increased, which was similar to the change pattern of NMDAR 1 expression. The AQP-1 mRNA expression in both 7 and $10 \mu \mathrm{A} \beta_{1-42}$ group was significantly higher compared with $0 \mu \mathrm{A} \beta_{1-42}$ group $(\mathrm{P}<0.01)$. The role of AQP-1 in the pathogenesis of $\mathrm{AD}$ will be one of the focuses in our future research. 
Further, the $10 \mu \mathrm{A} A \beta_{1-42}$ group was randomly divided into 3 subgroups: AD-NMDA, AD-MK-801, and AD-Ctrl group in order to study the association between NMDAR1 and APQ-1 expression in hippocampus tissues of AD rats. AQP-1 mRNA and protein expression in the 3 groups was compared by qRT-PCR and Western blot analyses. It was shown that APQ-1 mRNA and protein expression in AD-MK-801 group was markedly reduced compared with AD-Ctrl group, whereas APQ-1 expression in AD-NMDA was significantly increased, clearly suggesting that AQP-1 expression was regulated by the stimulation/inhibition of NMDAR1 expression. The two proteins might coordinately participate in the pathogenic process of AD.

\section{CONCLUSION}

In summary, this study has shown that expression abnormity of NMDAR1 is involved in the pathogenesis of AD. Further investigation on the mechanism of NMDAR1 in $A D$ rats suggested that NMDAR1 might regulate the pathogenic process through stimulating the expression of AQP-1. The current study will shed light on the pathogenesis of AD. Nevertheless, more in-depth studies will be needed in the future to validate our findings.

\section{REFERENCES}

1. Rafii MS, Aisen PS. Recent developments in Alzheimer's disease therapeutics. BMC Med. 2009;7:7.

2. Salloway S, Mintzer J, Weiner MF, Cummings JL. Disease-modifying therapies in Alzheimer's disease. Alzheimers Dement. 2008; 4(2):65-79.

3. Serizawa S, Chambers JK, Une Y. Beta amyloid deposition and neurofibrillary tangles spontaneously occur in the brains of captive cheetahs (Acinonyx jubatus). Vet Pathol. 2012;49(2):304-12.

4. Hardingham GE. Coupling of the NMDA receptor to neuroprotective and neurodestructive events. Biochem Soc Trans. 2009;37(Pt 6):1147-60.

5. Celso Constantino L, Tasca CI, Boeck CR. The Role of NMDA Receptors in the Development of Brain Resistance through Pre- and Postconditioning. Aging Dis. 2014; 5(6):430-41.

6. Tachibana N, Kinoshita M, Saito Y, Ikeda S. Identification of the N-Methyl-D-aspartate receptor (NMDAR)-related epitope, NR2B, in the normal human ovary: implication for the pathogenesis of anti-NMDAR encephalitis. Tohoku J Exp Med. 2013;230(1):13-6.

7. Saito Y, Tsuzuki K, Yamada N, Okado H, Miwa A, Goto F, Ozawa S. Transfer of NMDAR2 cDNAs increases endogenous NMDAR1 protein and induces expression of functional NMDA receptors in PC12 cells. Brain Res Mol Brain Res. 2003;110(2):15968.

8. Patil RV, Xu S, van Hoek AN, Rusinko A, Feng Z, May J, Hellberg M, Sharif NA, Wax MB, Irigoyen M, Carr G, Brittain T, Brown P, Colbert D, Kumari S, Varadaraj K, Mitra AK. Rapid Identification of Novel Inhibitors of the Human Aquaporin-1 Water Channel. Chem Biol Drug Des. 2016;87(5):794-805.

9. Soltani Z, Khaksari M, Shahrokhi N, Mohammadi G, Mofid B, Vaziri A, Amiresmaili S. Effect of estrogen and/or progesterone administration on traumatic brain injury-caused brain edema: the changes of aquaporin-4 and interleukin-6. J Physiol Biochem. 2016;72(1):33-44.

10. Foglio E, Rodella LF. Aquaporins and neurodegenerative diseases. Curr Neuropharmacol. 2010;8(2):112-21.

11. Hoshi A, Yamamoto T, Shimizu K, Ugawa Y, Nishizawa M, Takahashi H, Kakita A. Characteristics of aquaporin expression surrounding senile plaques and cerebral amyloid angiopathy in Alzheimer disease. J Neuropathol Exp Neurol. 2012;71(8):750-9.

12. Yang CC, Li L, Zhang L. Preparation and evaluation methods of commonly used animal models of Alzheimer disease. J. Chengdu Medical College .2013;8 (4): 1674-2257.

13. Hinrich AJ, Jodelka FM, Chang JL, Brutman D, Bruno AM, Briggs CA, James BD, 
Stutzmann GE, Bennett DA, Miller SA, Rigo F, Marr RA, Hastings ML. Therapeutic correction of ApoER2 splicing in Alzheimer's disease mice using antisense oligonucleotides. EMBO Mol Med. 2016;8(4):328-45.

14. Mortarino M, Franceschi A, Mancianti F, Bazzocchi C, Genchi C, Bandi C. Quantitative PCR in the diagnosis of Leishmania. Parassitologia. 2004;46(1-2):163-7.

15. Lu XY, Qi Q. Progresses in the researches on long-term synaptic potentiation mediated by N- methyl -D- aspartate receptors. Medical Recapitulate, 2011; 17(16):2424-6.

16. Nakanishi N, Tu S, Shin Y, Cui J, Kurokawa T, Zhang D, Chen HS, Tong G, Lipton SA. Neuroprotection by the NR3A subunit of the NMDA receptor. J Neurosci. 2009; 29(16):5260-5.

17. Luo L, Du YJ, Sun GJ. An investigation of A $\beta 140$, A $\beta 142$ and A $\beta 2535$ in an Alzheimer's disease model. Chinese Journal of Gerontology. 2014;9:2585-7.

18. Gordon MN, King DL, Diamond DM, Jantzen PT, Boyett KV, Hope CE, Hatcher JM, DiCarlo G, Gottschall WP, Morgan D, Arendash GW. Correlation between cognitive deficits and Abeta deposits in transgenic APP+PS1 mice. Neurobiol Aging. 2001; 22(3):377-85.

19. Kang DZ, Guo Z. Association of NMDAR1 expression and cognitive impairment in rats with brain injury. Chinese Journal of Clinical Neurosurgery 2010; 7 (03):119-122.

20. Oshio K, Watanabe H, Song Y, Verkman AS, Manley GT. Reduced cerebrospinal fluid production and intracranial pressure in mice lacking choroid plexus water channel Aquaporin-1. FASEB J. 2005;19(1):76-8. 